\title{
OBJETOS DE APRENDIZAGEM PARA ENSINO DE ESTRUTURAS DE DADOS FAZENDO USO DE REALIDADE AUMENTADA: SEDRA
}

\author{
LEARNING OBJECTS FOR TEACHING DATA STRUCTURES USING \\ AUGMENTED REALITY: SEDRA
}

Murilo Mickio Soares Hara, Robson Augusto Siscoutto

Universidade do Oeste Paulista - UNOESTE, Faculdade de Informática de Presidente Prudente - FIPP, Presidente Prudente, SP.

E-mail: murilo mickio@hotmail.com, robson@unoeste.br

RESUMO - O uso de Realidade Aumentada possibilita que a aplicação seja projetada no espaço do usuário, permitindo assim, uma maior interação, envolvimento e compreensão por parte do usuário, de forma mais natural, interativa e imersiva. Diante dessas possibilidades, a RA vem sendo empregada, cada vez, como tecnologia meio no ensino em diversas áreas do conhecimento. Os cursos de ciências da computação oferecem em sua matriz curricular diversos conteúdos focados na lógica, estruturas e programação. Elementos como como matrizes, vetores, pilhas, filas, árvores, dentre outras estudadas em Estrutura de Dados, são considerados abstratos e de difícil compreensão por parte dos discente. Diante disso, este trabalho apresenta o SEDRA (Sistema de Estrutura de Dados com Realidade Aumentada) que disponibiliza objetos de aprendizagem para estrutura de dados, fazendo uso de Realidade Aumentada e dispositivos móveis. Visando avaliar e quantificar a usabilidade da ferramenta por professores e profissionais, uma reinterpretação da heurística de Nielsen foi criada e aplicada.

Palavras-chave: realidade aumentada; objetos de aprendizagem; estrutura de dados.

\section{ABSTRACT -}

The use of Augmented Reality allows the application to be projected in the user's space, thus allowing a greater interaction, involvement and understanding on the part of the user, in a more natural, interactive and immersive way. Faced with these possibilities, RA has been increasingly used as a medium technology in teaching in several areas of knowledge. The courses of computer science offer in its curricular matrix diverse contents focused on the logic, structures and programming. Elements such as matrices, vectors, stacks, rows, trees, among others studied in Data Structure, are considered abstract and difficult to understand by students. Therefore, this work presents the SEDRA (Structure of Data Structure with Augmented Reality) that provides learning objects for data structure, making use of Augmented Reality and mobile devices. In order to evaluate and quantify the usability of the tool by

Recebido em:03/04/2017

Revisado em: 20/08/2017

Aprovado em: 04/10/2017 teachers and professionals, a reinterpretation of Nielsen's heuristics was created and applied.

Keywords: augmented reality; learning objects; data structure. 


\section{INTRODUÇÃO}

O uso de modelos e metodologias aplicadas no desenvolvimento da educação e aprendizagem, fazendo uso de tecnologias para auxílio em seus processos pedagógicos, já são uma realidade, dentre elas, podemos citar a Realidade Aumentada - RA, que, segundo Kirner e Siscoutto (2007), enriquece o ambiente real com objetos virtuais em tempo real, por meio de dispositivo tecnológico. Milgran (1994) também define RA como a mistura de mundos reais e virtuais em algum ponto da realidade/virtualidade contínua, que conecta ambientes completamente reais a ambientes completamente virtuais.

Focado em educação, a Realidade Aumentada vem cada vez mais sendo utilizada como objeto de aprendizagem, onde segundo Bettio e Martins (2004), citado por Audino e Nascimento (2012), qualquer entidade digital que tenha capacidade de exprimir algum conhecimento pode ser considerada objeto de aprendizagem. A aplicação da RA em estrutura de dados, com seus modelos, metodologias e representações gráficas podem contribuir para formas de aprendizados, uma vez que suas abstrações podem ser manipuladas interativamente para o ensino, possibilitando ao discente, resolver problemas ou aprender a matéria de diferentes formas. A falta de compreensão do raciocínio lógico, a forma de estudo dos alunos com a disciplina, geralmente memorizando a matéria e a falta de pré-requisitos em conteúdos relacionados, aliados com a falta de motivação do aluno, relacionamento com seus professores e suas didáticas/metodologias trazem certas dificuldades no aprendizado (SANTOS; COSTA, 2006). Logo, utilizar a tecnologia para ensino da própria tecnologia é bem cabível trazendo algo mais primoroso.

Diante dessas dificuldades, uma revisão bibliográfica foi desenvolvida e nenhuma ferramenta de Realidade Aumentada focada no ensino de Estruturas de Dados foi encontrada. Situação essa que demonstra a necessidade em buscar novas soluções que fortaleça e facilite o ensino e aprendizagem destes conteúdos.

Levando isso em consideração e atentando que a tecnologia vem como forma de potencialização e diferenciação para o aprendizado, este artigo apresenta uma ferramenta via estimular o aprendizado, especificamente, no ensino de estrutura de dados, fazendo uso de objetos de aprendizagem desenvolvidos em realidade aumentada interativa e para dispositivos móveis.

Dentre as principais contribuições alcançadas, pode-se citar:

- O aprendizado de estrutura de dados de uma maneira interativa e inovadora;

- Gera conhecimentos e conteúdos onde alunos e interessados poderão se aprofundar no assunto em decorrência deste projeto;

- Contribui para que aplicações afim sejam desenvolvidas, com intuito de ampliar a área de realidade aumentada para o ensino.

- Um método qualitativo e quantitativo para avaliar esse tipo de aplicação em um aspecto de usabilidade.

- Disponibiliza uma aplicação interativa para dispositivos móveis que faz uso de RA e objetos de aprendizagem para estudo de estruturas de dados.

$\mathrm{Na}$ seção 2 serão discutidos alguns trabalhos relacionados com foco no ensino, educação e seus efeitos esperados. Na seção 3 é discutida a ferramenta desenvolvida, destacando uma visão geral, arquitetura, recursos utilizados, metodologia e implementação. Na seção 4 é detalhado os testes de usabilidade realizadas, bem como seus resultados obtidos e discutidos. Por fim, na seção 5 são apresentadas as considerações finais e possíveis trabalhos futuros. 


\section{TRABALHOS RELACIONADOS}

Dentre as pesquisas encontradas, uma parte delas associa a área de Realidade Aumentada com Computação Móvel, denominada Realidade Aumentada Móvel (RAM) (AZUMA; BILLINGHURST; KLINKER, 2011). A RAM utiliza uma plataforma poderosa, onipresente e inteligente dos dispositivos móveis, uma vez que com a mobilidade é possível ter o acesso a informação de forma rápida, flexível, em tempo real e de qualquer lugar.

$\mathrm{Na}$ Realidade Aumentada os processos de construção de sistemas utilizam-se de dispositivos mais comuns, como uma webcam e marcadores impressos em papel, mais baratos e, devido a isso, mais acessíveis quando comparados aos sistemas de Realidade Virtual. Por este motivo, o crescente número de aplicações desenvolvidas em diferentes áreas que se utilizam desta tecnologia, tal como: medicina, entretenimento, engenharia, educação, entre outros tem sido notável.

No Brasil, sabe-se que já se existem diversas alternativas com o uso de Realidade Aumentada, demonstrando sua potencialidade no uso de ferramentas, principalmente na área de educação. Um dos exemplos bem práticos pesquisados foi 0 projeto LIRA (Livro interativo com realidade aumentada), que objetiva um produto informativo e didático subdivido em três temas: animais, meios de transporte e diversos, usando de recursos de som, imagem e textos. Implementado com o software ARToolkit que faz uso de marcadores fiduciais que estão associados a elementos dentro dos temas definidos. 0 livro é composto por páginas que possuem marcadores associado a objetos 3D. São disponibilizadas doze páginas, uma para cada objeto 3D. Apesar das conclusões apresentadas nos artigos não foram relatados resultados conclusivos (OLIVEIRA et al., 2006).

Outro projeto analisado foi o projeto SOL-RA, que representa um sistema solar interativo em Realidade Aumentada. Este trabalho foi desenvolvido com o suporte do Sistema de Autoria Colaborativa com Realidade Aumentada (SACRA) que é uma ferramenta de desenvolvimento de Realidade Aumentada, sem a necessidade de programação e ARToolKit. O SACRA utiliza pontos virtuais no ambiente, associados a cada um dos objetos 3D e sons, utiliza-se de recursos de imagens, animações, sons e interações com múltiplos marcadores do próprio SACRA sendo eles para reconhecimento do objeto, marcador "Inspeção" faz a ativação do objeto que se encontra no marcador de Referência, onde aparecerá o objeto 3D. O marcador "Controle" faz a mudança de um objeto que está sendo visualizado pelo próximo. O marcador "Cópia" duplica o objeto. O marcador "Transporte" leva o objeto para qualquer área dentro do campo de visualização do webcam. O marcador "Apagador" apaga os objetos, quando acontece a colisão entre sua esfera e a esfera do objeto que se quer apagar. O marcador "Status" mostra alguns dados importantes do objeto, como a distância do objeto ao ponto central, visando facilitar o conhecimento no contexto da estrutura e comportamento do sistema solar. Este projeto foi relatado como principal contribuição à integração de recursos e sua utilização para fornecer informações sobre o Sistema Solar. Complementarmente, buscou-se apoiar o usuário na manipulação do sistema, facilitando a utilização nas fases iniciais (OKAWA; KIRNER; KIRNER, 2010).

O RExLab - UFSC, é uma aplicação da Realidade Aumentada para Simulação de Experimentos Físicos em Dispositivos Móveis desenvolvido em java com tecnologia QCAR (Qualcomm Augmented Reality) para dispositivos android com biblioteca OpenGL, Blender e android SDK, também se utilizando de marcadores para renderização do objeto 3D, na área de experimentos físicos simulando situações. A aplicação usa a tela do aparelho como uma lente onde é apresentado o ambiente real aumentado com os objetos virtuais 3D. O processamento 
da imagem da câmera e a inserção dos modelos virtuais 3D acontece em tempo real. Sendo possível a interação do usuário com o experimento através do dispositivo, mudando o estado do experimento e podendo realizar todas as simulações possíveis do mesmo experimento em um ambiente real. Foi realizada a modelagem do experimento quadro elétrico e sua texturização de acordo com seus diferentes estados possíveis, por se tratar como uma melhor opção para modelagem em 3D e uma maior facilidade para simular suas mudanças de estado. Apesar de não terem sido modelados todos os experimentos a literatura aponta bons resultados pelo seu objetivo que era de fornecer uma integração maior dos alunos com o aprendizado e fornecendo meios alternativos de transmissão de conhecimento. Seus testes foram feitos simulando seus estados (MALBOS, 2014).

Foi encontrada também um trabalho baseada em jogos de realidade aumentada no ensino de sólidos geométricos, cujo nome da aplicação "Vertice", foi o desenvolvimento de um jogo com realidade aumentada para o ensino da geometria com uso da engine unity3D juntamente com Vuforia e Blender, e conta com um painel com o marcador (padrão de reconhecimento), que indica as coordenadas onde se desenrola o jogo, por fim, a construção efetiva da aplicação de base educacional com elementos de jogo, através de realidade aumentada com um design focado no utilizador e nos objetivos. Foram feitos experimentos através de duas turmas do ensino básico com a disponibilização da aplicação a apenas uma. Por fim, foi apresentado um teste às duas turmas para validação da proposta, resultando na capacidade de despertar curiosidade, motivação e iniciativa nas crianças, devido ao seu caráter envolvente e interativo. Do ponto de vista pedagógico, ajudou as a alcançar as competências que o jogo visava desenvolver: capacidade de visualização; a compreensão de propriedades dos sólidos geométricos e a familiarização com o vocabulário da geometria. Ainda segundo Leitão (2013), o fato dos smartphones serem portáteis e com o recente aprimoramento tecnológico eles tornaram-se presentemente uma plataforma muito desejável para o desenvolvimento de soluções com realidade aumentada.

Outra aplicação interessante é o Augmented Chemistry, desenvolvido com uso de ARToolKit, e que faz uso da Realidade Aumentada a partir de interfaces tangíveis para auxiliar no ensino da química (FJELD; VOEGTLI, 2002). O aluno, utiliza uma espécie de garra para pegar elementos da tabela periódica de um catálogo específico, contendo um elemento por página, e assim vai montando a molécula desejada, que pode ser visualizada através de um monitor. Para movimentação do objeto, o aplicativo conta com um cubo, que ao ser girado, movimenta a molécula, dando ao mesmo uma visão de qualquer ângulo da substância. Entretanto, o sistema dificulta o trabalho colaborativo, uma vez que cada estudante deverá utilizar seu próprio material.

Finalmente, não foi encontrado trabalhos que se remetesse a Realidade Aumentada no ensino da Estrutura de Dados, alvo deste trabalho.

\section{PROJETO E IMPLEMENTAÇÃO}

Esta seção apresenta o projeto e a implementação do sistema SEDRA, destacando o processo, a arquitetura, os recursos, os métodos, a interface e a usabilidade utilizados no desenvolvimento. Na seção 3.1 é discutida a arquitetura do sistema e o cenário atual onde a projeto será realizado destacando os problemas encontrados, seus componentes e objetos de uso. Na 3.2 será descrito toda a construção, interface e usabilidade da ferramenta.

\subsection{Arquitetura}

A figura 1 apresenta a arquitetura do SEDRA que está dividida em 4 módulos, são eles: Technologies, Visualization, Engine e Database. 
Figura 1. Arquitetura SEDRA

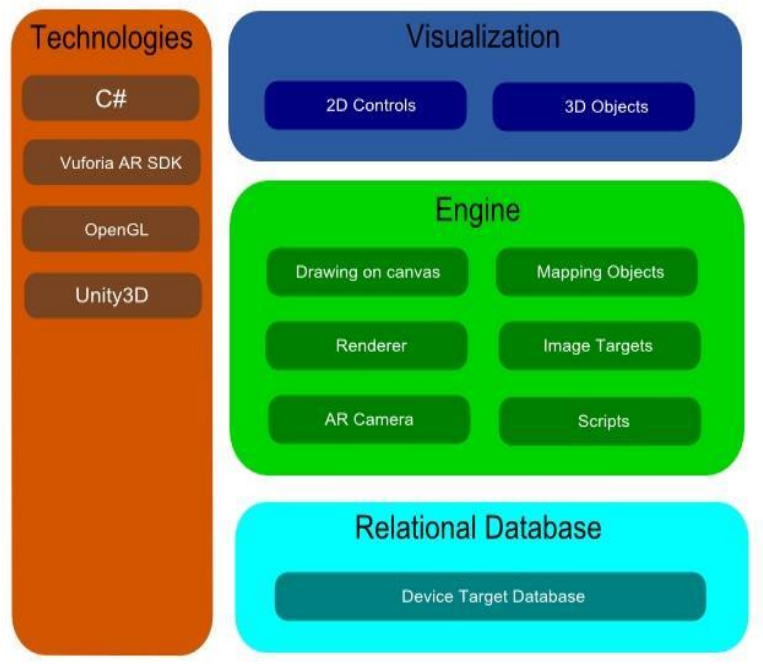

Fonte: O Autor.

O Módulo Technologies destaca os recursos utilizados no desenvolvimento da aplicação. A Unity3D se destaca por ser um motor de jogo amplamente utilizado e atualizado, disponibilizando duas linguagens de programação: C\# e JavaScript. Neste projeto foi utilizada a linguagem $\mathrm{C \#}$ bem como o SDK Vuforia que é uma biblioteca para uso de Realidade Aumentada em celulares. Por fim, a linguagem OpenGL foi utilizada no unity como seu motor de renderização.

O módulo Visualization, disponibiliza botões de controle para a animação dos objetos 3D, permitindo que o usuário controle o estado da animação. Além de permitir a interação direta com os objetos 3D na tela, este módulo se divide em: $2 D$ Controls e 3D Objects. O 2D Controls é responsável por adicionar funcionalidades ligadas à simulação como play, reset, pause, dentre outros, fornecendo a visibilidade dos eventos. O 3D Objects é a área responsável pelo campo de interatividade com os objetos em cena permitindo seu toque para mudança de posição e tamanho.

O Módulo Engine consiste nos recursos e algoritmos usados para a criação e manipulação dos dados utilizados as estruturas de dados criadas. Este módulo é composto por:
- Mapeamento dos objetos: define os objetos que serão sobrepostos sobre a target;

- Drawing on canvas: cenário onde são mapeados os objetos 2D de controle;

- Renderer: motor de renderização da aplicação, que no caso do unity3D é o OpenGL

- Image Targets: responsável por receber os objetos da cena e reconhecimento para qual objeto será mostrado, além de prover o efeito de Realidade Aumentada. Recurso do Vuforia;

- AR Câmera: recurso do Vuforia para prover da Realidade Aumentada;

- Scripts: códigos C\# utilizados na aplicação.

O Módulo Relational Database é responsável por armazenar os dados de eventos e da aplicação, que no caso se dá no próprio aparelho Mobile.

\subsection{Desenvolvimento}

O SEDRA utiliza como marcadores imagens pré-definidas ao invés de marcadores fiduciais. Por meio do Vuforia é possível mapear imagens e transformá-las em marcadores para que os objetos 3D usem como apoio para então se obter a experiência de RA. Diante disso, foi escolhido o livro "Introdução a Estrutura de Dados Com técnicas de programação em C" do Waldemar Celes, Renato Cerqueira e José 
Lucas Rangel, na área do tema tratado pelo fato de conter figuras mais comuns e simplistas da matéria tratada e além de ser de literatura básica para muitas universidades.

Os marcadores utilizados no projeto foram definidos com base nas imagens das estruturadas de dados disponíveis no livro, conforme figura 2. Foram escolhidas quatro estruturas básicas como imagens que seriam usadas como marcadores, sendo elas: pilha, fila, lista e árvore binária.

Figura 2. Livro Base e exemplo de marcador do SEDRA.

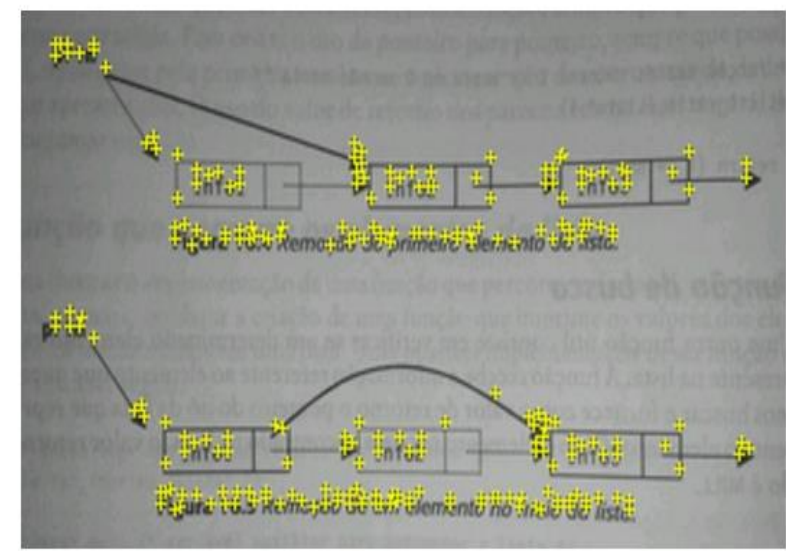

Fonte: O Autor

Com o marcadores definidps, estes deverão ser importados para a plataforma online do Vuforia mediante um registro e criação da base de dados, onde a imagem do marcador é processada para descarregarmos o ficheiro (*. unitypackage) e importá-los para o Unity3D. Já no ambiente Unity é necessária a instalação da extensão do Vuforia para Unity disponível em Vuforia (2017). Após a importação do Vuforia, este criará um conjunto de pastas dentro do projeto com seus respectivos componentes.

Depois de todo o processo de preparação do ambiente, é iniciado a sua configuração. Primeiramente, a câmara principal é substituída pelo componente "AR Câmera" encontrada no diretório VuforiaPrefabs, após este processo coloca-se em cena o componente Image Target também encontrado no diretório Prefabs, este será usado como marcador. Com a AR Câmera selecionada, foram configuradas em suas propriedades, a chave de licença adquirida no cadastro do Vuforia e habilitados no campo "Datasets" os dois checkboxes "Load Database" e "Activate". Do mesmo modo, com o "Image Target" em cena selecionado, em suas propriedades no campo script "Image Target Behaviour" é necessário configurar o componente no campo Data Set com o marcador que pretendemos usar, fazendo com que este apareça na tela no componente Image Target posto em cena. Deste ponto em diante, o processo de modelagem começou a ser desenvolvido como mostrado na figura 3 , bem como as animações, posicionamento dos objetos, scripts e telas utilizando se de componentes do próprio Unity como: Cubo, Esfera, Cilindro e Text 3D. 
Figura 3. Configuração e Modelagem das estruturas do SEDRA

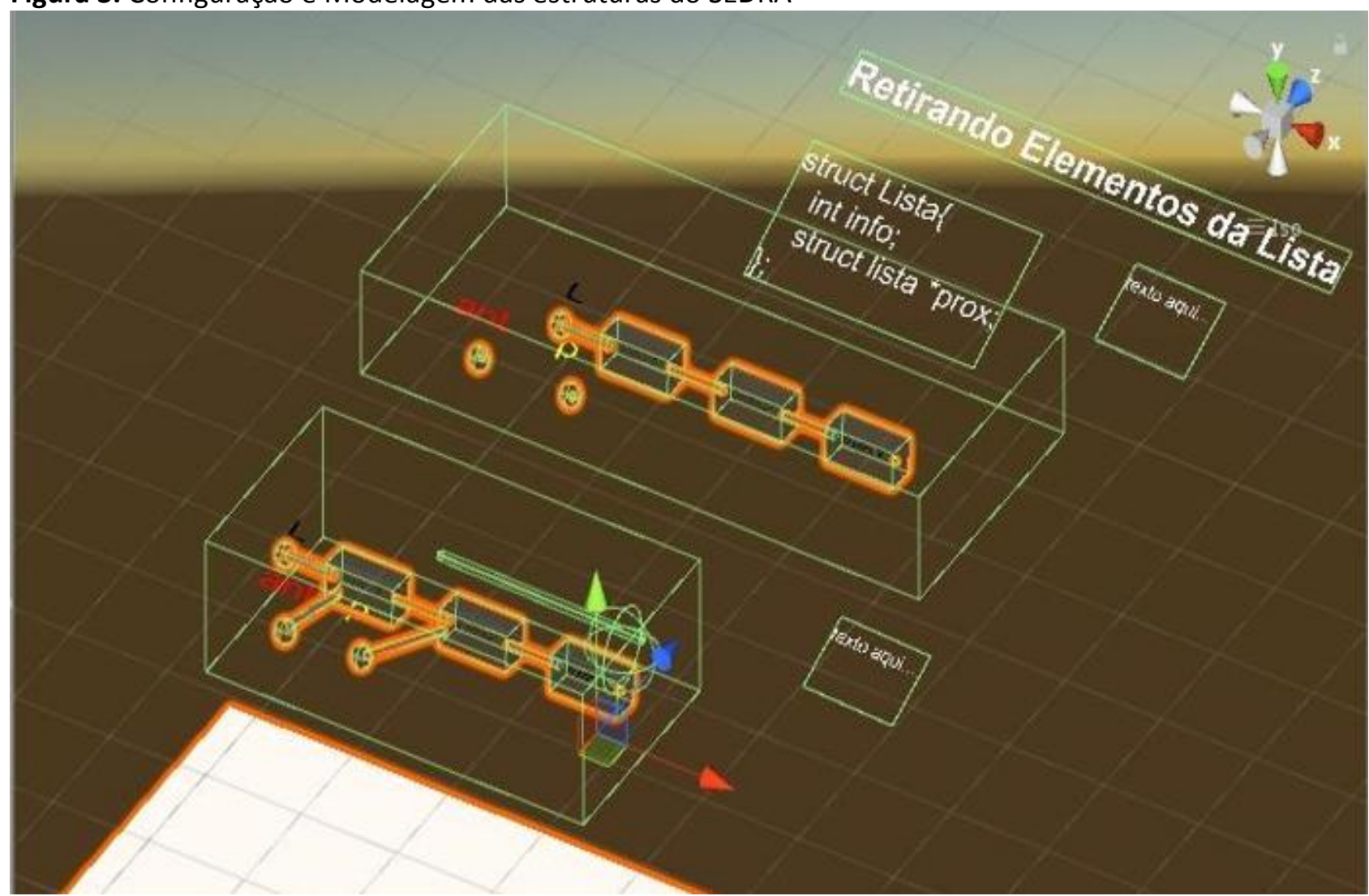

Fonte: O Autor

As animações consistem em operações básicas nas estruturas de dados, como por exemplo: efetuar a inserção de algum elemento na estrutura selecionada, posição onde iriam se manter os objetos e para onde iriam posteriormente, para isso foi utilizado o componente "Animation" e "Animator" (Fig.4). O "Animation" representava as animações criadas a partir da movimentação dos objetos e gravação de seus frames, gerando um arquivo (*.anim). 0 "Animator" (*.controller), eram os controladores, que a partir de um conjunto de "Animation" era possível seu controle e gerenciamento de prioridade de processo ou seja qual animação seria posta primeiro, ou segundo. Sendo feito este processo para todos os objetos e suas "Image Targets". 
Figura 4 - Animação SEDRA

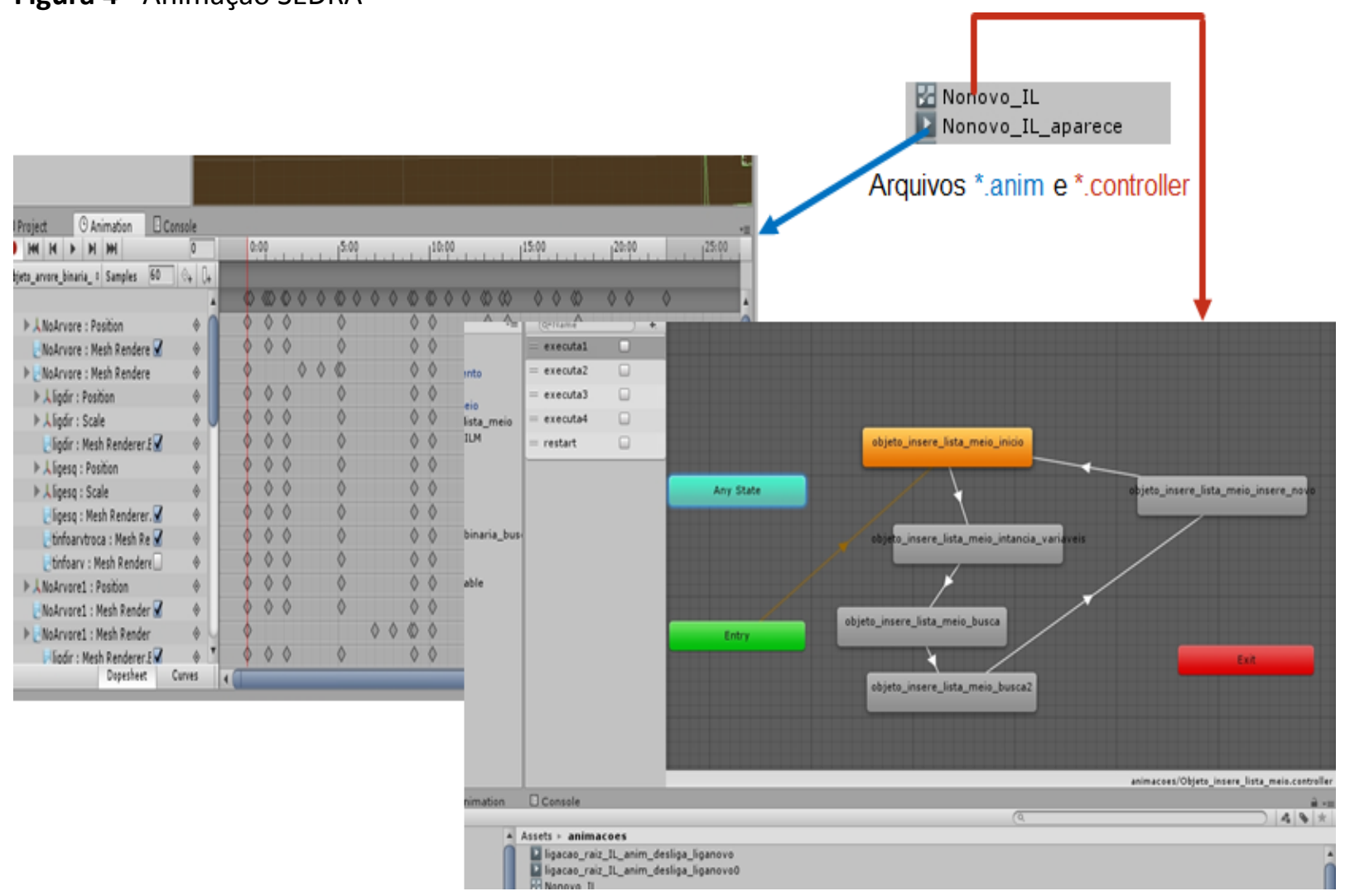

Fonte: O Autor.

Para a representação textual foram criados scripts que executam e apresentam as animações no momento correto. Trechos de códigos que manipulam o exemplo em execução são apresentados na tela com cor diferenciada, além de scripts responsáveis pelo movimento e aumento do zoom dos objetos.

A interface da aplicação possui uma tela inicial (Figura 5), utilizando-se dos componentes "Canvas" no Unity3D para produção dos botões de controle e ajuda.

Figura 5. Projeto das interfaces

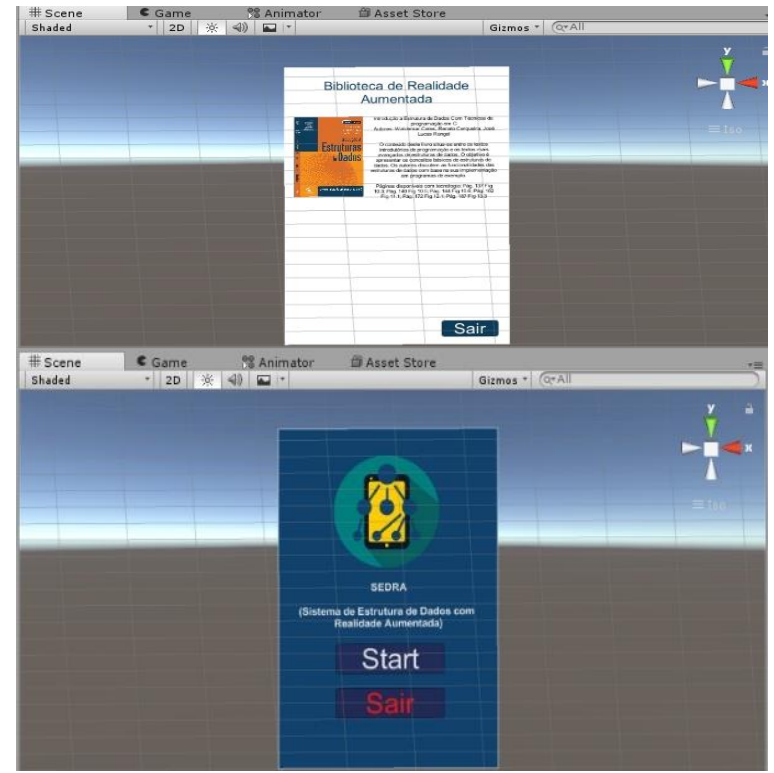


Fonte: O Autor.

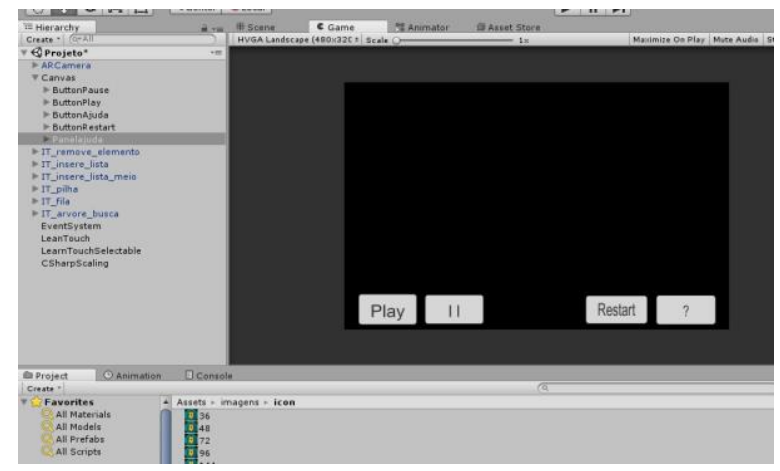

\subsection{Interface Gráfica e Usabilidade}

Ao executar o SEDRA, o usuário será direcionado para sua tela inicial, conforme a figura 6. Ao apertar "Start" este será direcionado para a interface "Biblioteca do SEDRA" onde é mostrado o livro e algumas descrições sobre a tecnologia de Realidade
Aumentada, bem como as páginas sobre a tecnologia implementada e suas figuras. Foi projetado desta forma com o pressuposto de que mais adiante pudesse haver outras literaturas incluídas no aplicativo.

Figura 6. Tela Inicial e Biblioteca do SEDRA.

Fonte: O Autor.

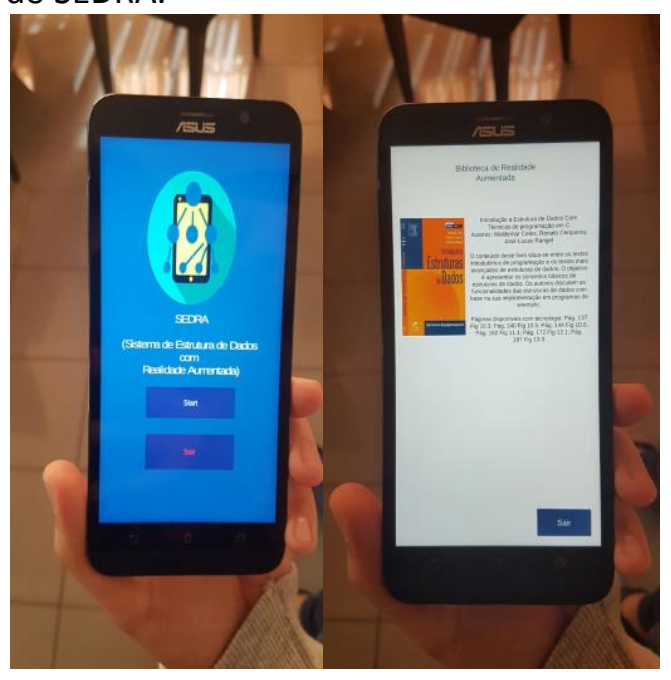

Clicando sobre o livro desejado, é disponibilizada a interface para a experiência de Realidade Aumentada. Com o livro base em mãos é só apontar a câmera para as imagens cadastradas como marcador e clicar no botão "Play" para começar a simulação. $O$
SEDRA possui uma usabilidade simplificada, contando com um botão de ajuda para que o aluno não precise de muito treinamento para uso, conforme figura 7. 
Figura 7. Imagens do SEDRA

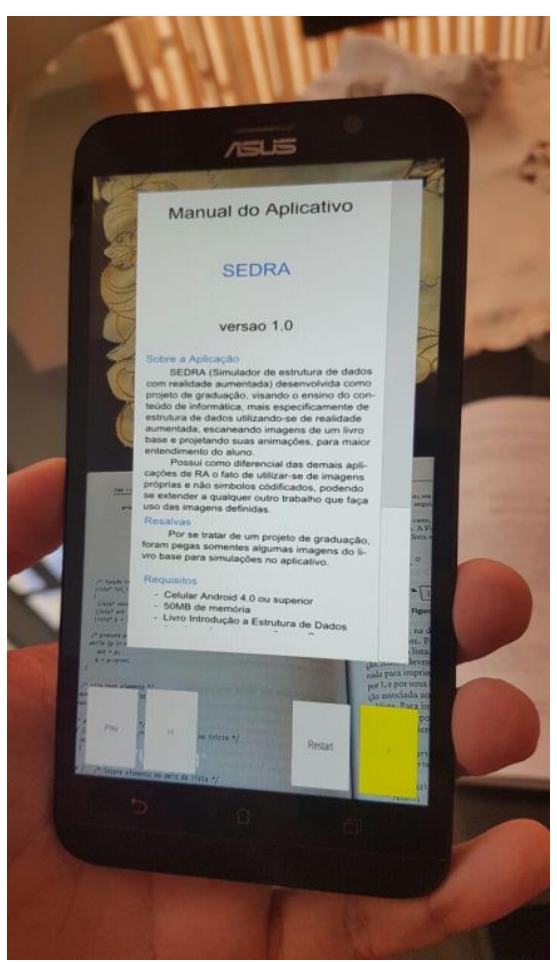

Fonte: O Autor.

A interface conta com eventos de toque de pincelagem em zoom e tocar e arrastar para que mediante uma tela menor o usuário possa alterar a dimensão e posição dos objetos 3D, bem como melhorar a visualização. Além disso, é permitido o modo Portraid e Landscape. Seus botões de controle permitem que as animações sejam executadas e paradas no momento desejado. $\mathrm{O}$ uso dos gestos comuns de zoom e translação permitem o ajuste dos objetos na tela para melhor visualização e acompanhamento da animação. Texto de apoio como o nome da estrutura e códigos diferenciados por cores para cada objeto são apresentados (a cor corresponde ao trecho de código que está sendo processado). A cor laranja é a padrão para representar operações, conforme se pode visualizar na figura 8. 
Figura 8. Imagens do SEDRA.

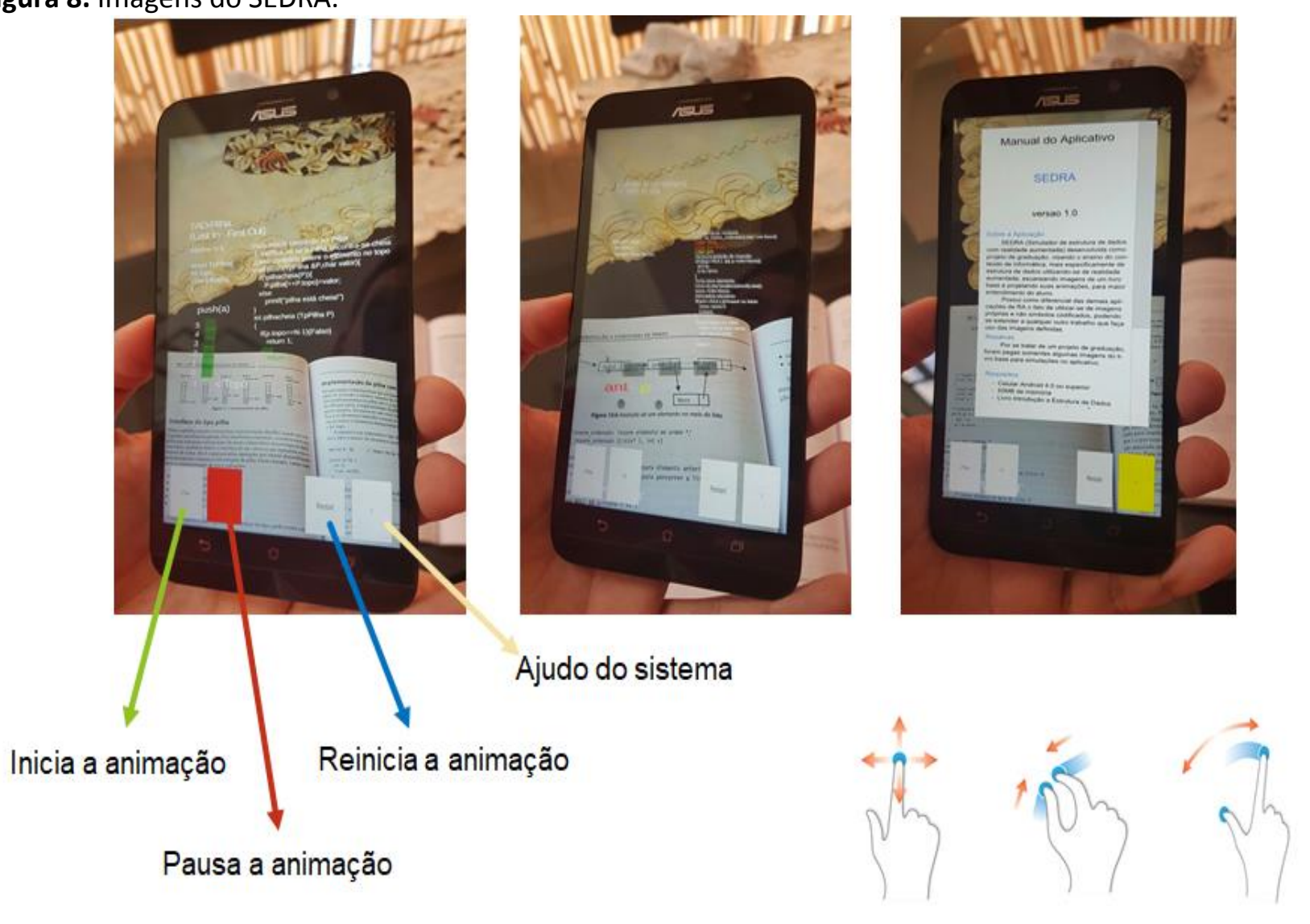

Fonte: O Autor.

\section{TESTES E RESULTADOS}

Algumas técnicas de avaliação para testes de usabilidade podem incluir uma lista de métodos que direciona os esforços dos usuários em realizar uma variedade de tarefas em um protótipo ou sistema, estas tarefas são observadas por inspetores que coletam dados referentes aos processos de interação do usuário, incluindo erros cometidos pelo usuário, quando e onde eles confundem-se ou se frustram, a rapidez com a qual o usuário realiza a tarefa, se eles obtêm sucessos na realização da tarefa e a satisfação do usuário com a experiência.

Neste projeto, o objetivo da aplicação deste teste foi para entender a opinião do usuário sobre a aplicação, tanto em questões mais gerais como em questões mais específicas.

O teste foi aplicado para professores especialistas em usabilidade. Os avaliadores convidados, individualmente, a participar do teste, utilizaram um celular e o livro físico base da aplicação dentro de um tempo de, aproximadamente, 25 minutos. Durante o teste havia um moderador (pessoa a aplicar o teste) que realizou a explicação do teste e fez uma introdução sobre a aplicação SEDRA por cerca de 5 minutos.

Foi preparado um material com um texto introdutório (carta de apresentação Figura 3), um questionário de pré-teste (Tabela 1) e um questionário de pós-teste (Tabela 2), contendo as questões a serem avaliadas.

Depois, por cerca de 2 minutos, os avaliadores responderam ao questionário de pré-teste apresentado na Tabela 1 Resultado do Pré-Teste - SEDRA. 
Tabela 1. Resultado do Pré-Teste

\begin{tabular}{|c|c|c|c|c|c|}
\hline Perfil do Usuário - Pré-Teste & Avaliador 1 & $\begin{array}{l}\text { Avaliador } \\
2\end{array}$ & $\begin{array}{c}\text { Avaliador } \\
3\end{array}$ & $\begin{array}{c}\text { Avaliador } \\
4\end{array}$ & $\begin{array}{l}\text { Avaliado } \\
\text { r } 5\end{array}$ \\
\hline \multicolumn{6}{|l|}{ 1: Informações pessoais } \\
\hline 1.1 Sexo: ( ) M () F & $\mathrm{F}$ & $\mathrm{M}$ & $M$ & $\mathrm{~F}$ & $M$ \\
\hline 1.2 Idade: & 42 & 41 & 32 & 40 & 41 \\
\hline $\begin{array}{l}1.3 \text { Nível de escolaridade: ( ) } \\
\text { Graduação ( ) Pós-Graduação ( ) } \\
\text { Mestrado ( ) Doutorado }\end{array}$ & $M$ & $\mathrm{D}$ & $M$ & $M$ & $\mathrm{M}$ \\
\hline \multicolumn{6}{|l|}{$\begin{array}{l}\text { 2: Como você classifica o uso de } \\
\text { ambientes colaborativos? }\end{array}$} \\
\hline $\begin{array}{l}\text { ( ) funciona de maneira incrivelmente } \\
\text { boa }\end{array}$ & & $x$ & & & \\
\hline ( ) funciona bem & $\mathrm{X}$ & & $\mathrm{X}$ & $\mathrm{X}$ & $\mathrm{X}$ \\
\hline \multicolumn{6}{|l|}{ ( ) funciona regularmente } \\
\hline \multicolumn{6}{|l|}{ ( ) funciona de maneira deplorável } \\
\hline \multicolumn{6}{|l|}{ ( ) nunca utilizei } \\
\hline \multicolumn{6}{|l|}{$\begin{array}{l}\text { 3: Como você classifica o uso de } \\
\text { sistemas de mapas digitais com } \\
\text { eventos meteorológicos? }\end{array}$} \\
\hline \multicolumn{6}{|l|}{$\begin{array}{l}\text { ( ) funciona de maneira incrivelmente } \\
\text { boa }\end{array}$} \\
\hline ( ) funciona bem & & $\mathrm{X}$ & $\mathrm{X}$ & & \\
\hline ( ) funciona regularmente & $\mathrm{X}$ & & & $\mathrm{X}$ & \\
\hline \multicolumn{6}{|l|}{ ( ) funciona de maneira deplorável } \\
\hline ( ) nunca utilizei & & & & & $x$ \\
\hline \multicolumn{6}{|l|}{$\begin{array}{l}\text { 4: Como você classifica o uso de } \\
\text { sistemas de mapas digitais integrados } \\
\text { com ambientes colaborativos? }\end{array}$} \\
\hline \multicolumn{6}{|l|}{$\begin{array}{l}\text { ( ) funciona de maneira incrivelmente } \\
\text { boa }\end{array}$} \\
\hline \multicolumn{6}{|l|}{ ( ) funciona bem } \\
\hline ( ) funciona regularmente & & & & $\mathrm{X}$ & $\mathrm{X}$ \\
\hline \multicolumn{6}{|l|}{ ( ) funciona de maneira deplorável } \\
\hline ( ) nunca utilizei & $\mathrm{X}$ & $x$ & $\mathrm{X}$ & & \\
\hline
\end{tabular}

Para o questionário pós-teste foram utilizadas 12 questões referentes à usabilidade com cinco alternativas possíveis, de acordo com a escala Likert de cinco pontos ("Concordo Totalmente - 5", "Concordo - 4", "Indeciso - 3", "Discordo - 2" e "Discordo Totalmente - 1"), a fim de medir o nível de concordância à afirmação. As questões foram criadas tendo como base as reinterpretações de Nielsen (1994). Após a explicação sobre o SEDRA e a resposta do pré-teste, os avaliadores puderam experimentar a aplicação por 10 minutos cada. O pós-teste foi respondido logo após e durou em torno de 5 minutos. A Tabela 2 apresenta a média das opiniões dos avaliadores em relação a aplicação SEDRA. 
Tabela 2. Questionário Pós-Testes x Heurísticas X Média Obtida

\begin{tabular}{|c|c|c|}
\hline Questões & Heurística & $\begin{array}{l}\text { Média das } \\
\text { notas } \\
1 \text { a } 5 \\
\end{array}$ \\
\hline $\begin{array}{l}\text { 1-Para mim foi fácil entender como utilizar a aplicação e } \\
\text { qual era o objetivo. }\end{array}$ & $\begin{array}{l}\text { Facilidade de } \\
\text { uso }\end{array}$ & 4,4 \\
\hline $\begin{array}{l}\text { 2-Quando existiam mensagens na aplicação, sejam textuais } \\
\text { ou em vídeo, a linguagem era entendida facilmente. }\end{array}$ & $\begin{array}{l}\text { Prevenção de } \\
\text { erros }\end{array}$ & 3,2 \\
\hline $\begin{array}{l}\text { 3-A forma de interação, navegação e representação é } \\
\text { compatível como o mundo real e/ou padronizados entre os } \\
\text { usuários. }\end{array}$ & $\begin{array}{l}\text { Compatibilidade do } \\
\text { Sistema com o } \\
\text { mundo } \\
\text { real }\end{array}$ & 4,0 \\
\hline $\begin{array}{l}\text { 4-Em nenhum momento eu me senti perdido na aplicação, } \\
\text { sem saber o que fazer. }\end{array}$ & $\begin{array}{c}\text { Visibilidade } \\
\text { do status do } \\
\text { sistema }\end{array}$ & 3,4 \\
\hline $\begin{array}{l}\text { 5-Os símbolos (ligações, por exemplos) foram posicionados } \\
\text { de forma correta na aplicação. }\end{array}$ & Precisão & 4,2 \\
\hline $\begin{array}{l}\text { 6-Eu sempre executava as mesmas ações para acionar as } \\
\text { mesmas funcionalidades }\end{array}$ & $\begin{array}{l}\text { Consistência } \\
\text { e padrões }\end{array}$ & 4,2 \\
\hline $\begin{array}{l}\text { 7-Somente me foi apresentado na interface informações } \\
\text { (símbolos, por exemplo) apropriados, sem poluir a interface. }\end{array}$ & $\begin{array}{c}\text { Estética e } \\
\text { design minimalista }\end{array}$ & 3,6 \\
\hline 8-O sistema disponibilizou um material de ajuda de uso. & $\begin{array}{c}\text { Ajuda e } \\
\text { Documentação }\end{array}$ & 4,4 \\
\hline 9-O Ajuda foi útil e fácil de entender? & $\begin{array}{c}\text { Ajuda e } \\
\text { Documentação }\end{array}$ & 4,2 \\
\hline $\begin{array}{l}\text { 10-A interface foi projetada de tal forma que usuário } \\
\text { consegue utilizar a aplicação de forma fácil, ou seja, exige } \\
\text { pouco treinamento. }\end{array}$ & $\begin{array}{l}\text { Reconhecimento ao } \\
\text { invés de } \\
\text { relembrança }\end{array}$ & 3,8 \\
\hline 11-Foi uma experiência agradável utilizar a aplicação. & Satisfação & 4,0 \\
\hline 12-Eu utilizaria novamente a aplicação, se fosse possível. & Satisfação & 4,4 \\
\hline & Média Total & 3,9 \\
\hline
\end{tabular}

Todas as questões tiveram o mesmo peso e o SEDRA atingiu a média total de 3,9 (de 0 a 5) o que se considera um resultado bom. $O$ gráfico 1 apresentam as respostas de cada avaliador para cada questão do teste de usabilidade do SEDRA. 
Gráfico 1. Respostas dos avaliadores do SEDRA

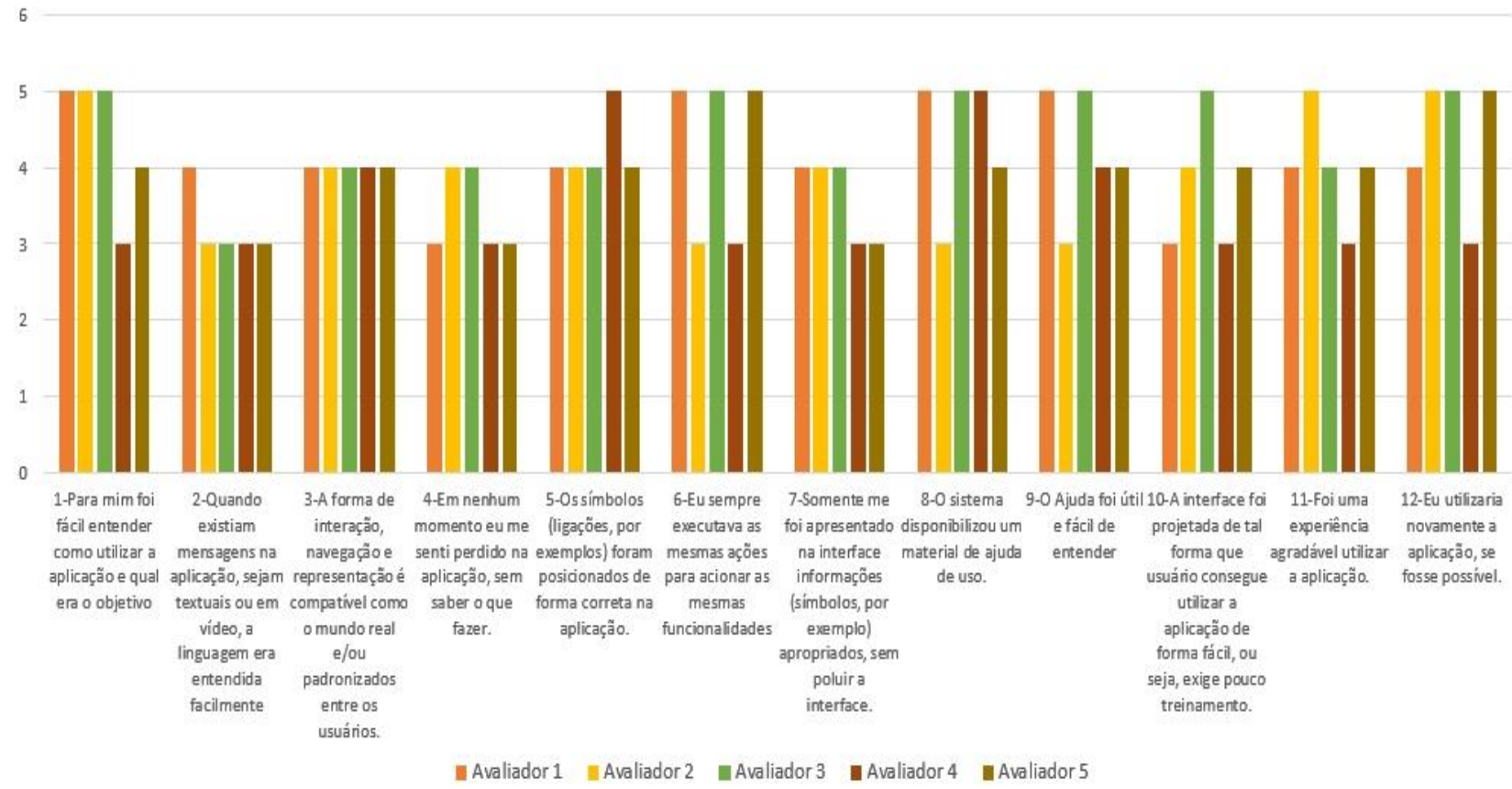

Fonte: O Autor.

\subsection{Discussão dos Resultados}

O objetivo deste projeto teve como intuito estudar e implementar objetos de aprendizagem em realidade aumentada para conteúdo de estrutura de dados para dispositivos móveis. A escolha desta plataforma se dá pelo fato de ser uma plataforma livre e sem implicar em custos com equipamentos suplementares e pelo fato de hoje em dia o smartphone ser umas das tecnologias mais utilizáveis e talvez até mais acessíveis por qualquer camada social.

Em relação às tecnologias utilizadas e softwares utilizados na criação do jogo destacaram-se pela facilidade de acesso à informação. Tanto o Unity3D como o Vuforia possuem um extenso conjunto de fontes de informação como tutoriais em vídeo (tanto no canal Youtube, como nos seus próprios websites) e comunidades que ajudam em muito na obtenção do conhecimento. Já na escolha do tema, foi de principal importância o fato de, por se tratar de uma tecnologia bem relevante nos dias atuais, podendo trazer muitos benefícios para a sociedade em geral e está sendo muito utilizado em diversos meios, ainda não se tinham relatos de aplicações de ensino para estrutura de dados com o uso de Realidade Aumentada. Em vista de que se trata de uma área de difícil entendimento por parte do aluno.

$O$ processo de desenvolvimento requereu o estudo da plataforma utilizada bem como sua biblioteca e do tema proposto. Foi encontrado outras dificuldades pelo fato do tema tratado, pois este é abstrato e não possui um padrão universal em sua estrutura, as questões do pós-teste aplicados de número 2, 4 e 7 que tratam da interface do SEDRA mostram que ainda é preciso melhorias na visualização dos objetos. Isso se dá pelo fato de ter que adaptar todo o conteúdo de forma que fosse possível a percepção em telas menores permitindo compreender que as plataformas móveis nem sempre têm uma eficiência que permita um sistema para gerir toda a informação, mesmo o aplicativo contando com a possibilidade de mudar e aumentar os objetos de acordo com o que usuário deseja, mas ao mesmo tempo, o avanço tecnológico permite cada vez mais uma evolução positiva neste sentido. No geral o SEDRA teve boas notas atingindo uma média de 3.9 na escala de 5 . 


\section{CONSIDERAÇÕES FINAIS E TRABALHOS FUTUROS}

A Realidade Aumentada se mostra ser um campo em grande crescimento podendo ser explorado para diversas aplicações adaptadas a cada área de conhecimento, com grande potencial na área da educação. $O$ SEDRA demonstrou despertar a motivação e curiosidade por parte dos usuários, contudo mostrou também que ainda é possível melhorias em termos de usabilidade para adaptação e melhor entendimento do aluno.

Como possíveis trabalhos futuros atrelado com outras tecnologias, como por exemplo o uso de som para alunos com deficiência auditiva e até mesmo por meio de um jogo interativo estimulando melhor a interação com o usuário. A construção do simulador abre caminhos para desenvolvimentos futuros, em cima do tema estrutura de dados, para outras plataformas, e até mesmo a continuação para demais livros focados no tema. Academicamente, o desenvolvimento deste projeto poderá abrir uma grande gama de estudos na universidade e também outros alunos poderão se aprofundar no assunto em decorrência deste projeto desenvolvido.

\section{REFERÊNCIAS}

AUDINO, D.F.; NASCIMENTO, R.S. Objetos de aprendizagem - diálogos entre conceitos e uma nova proposição aplicada à educação. Revista Contemporânea de Educação, v. 5, n. 10, fev. 2012. ISSN 1809$5747 . \quad$ Disponível em: <https://revistas.ufri.br/index.php/rce/article Liew/1620/1468>. Acesso em: 16 Jun. 2017.

AZUMA, R.; BILLINGHURST, M.; KLINKER, G. Editorial: Special Section on Mobile Augmented Reality. Computer Graphics, pp. vii-viii. 2011.

https://doi.org/10.1016/i.cag.2011.05.002

FJELD, M.; VOEGTLI, B.M. Augmented chemistry: An interactive educational workbench. In: Mixed and Augmented Reality, 2002. ISMAR 2002. Proceedings.
International Symposium on. IEEE, 2002. p. 259-321.

KIRNER, C.; SISCOUTTO, R. Realidade virtual e aumentada: conceitos, projeto e aplicações. In: SYMPOSIUM ON VIRTUAL AND AUGMENTED REALITY, 9. Petrópolis: SBC, 2007.

LEITÃO, R. Aprendizagem baseada em jogos: realidade aumentada no ensino de sólidos geométricos. 2013. Dissertação (Mestrado) Universidade Aberta, 2013.

MALBOS, A. N. A. et al. Aplicação da Realidade Aumentada para simulação de experimentos físicos em dispositivos móveis. In: REMOTE ENGINEERING AND VIRTUAL INSTRUMENTATION (REV), INTERNATIONAL CONFERENCE ON IEEE, 11. Proceedings... 2014. p. 231-235.

MILGRAN, P. et. al. Augmented Reality: A Class of Displays on the Reality-Virtuality Continuum. Telemanipulator and Telepresence Technologies, SPIE, v.2351, p. 282-292, 1994. https://doi.org/10.1117/12.197321

NIELSEN, J. Usability engineering. Elsevier, 1994.

OKAWA, E.S.; KIRNER, C.; KIRNER, T.G. Sistema solar com realidade aumentada. In: WORKSHOP DE REALIDADE VIRTUAL E AUMENTADA-WRVA, 7. Anais... 2010. p. 7276.

OLIVEIRA, F.C. et al. Projeto LIRA-Livro Interativo com Realidade Aumentada. In: WORKSHOP EM INFORMÁTICA NA EDUCAÇÃO - SBIE, SIMPÓSIO BRASILEIRO DE INFORMÁTICA NA EDUCAÇÃO - SBIE, 7. Anais... UNB/UCB, 2006.

SANTOS, R. P.; COSTA, H. A. X. Análise de Metodologias e Ambientes de Ensino para Algoritmos, Estruturas de Dados e Programação aos iniciantes em Computação e Informática. INFOCOMP Journal of 
Computer Science, v.5, n.1, p.41-50, mar. $2006 . \quad$ Disponível em: $<$ http://www.dcc.ufla.br/infocomp/index.ph $\mathrm{p} /$ INFOCOMP/article/view/121>. Acesso em: 16 Jun. 2017.

UNITY. Unity Technologies. Unity 4. 2013. Disponível em: <http://unity3d.com/unity/>. Acesso em: 18 Jun. 2017.

VUFORIA. Vuforia tutorials. 2017. Disponível em:

$<$ http://developer.vuforia.com/resouces/tuto rials>. Acesso em: 18 Jun. 2017. 